\title{
Limiar anaeróbico (V4) e frequência cardíaca de cavalos Crioulos condicionados para prova funcional
}

\author{
[Anaerobic threshold (V4) and heart rate of Crioulo horses conditioned for functional testing]
}

\author{
L.A. Amaral, A. Torres, V. Rabassa, C. F. Martins, M. N. Correa, C.E.W. Nogueira
}

Universidade Federal de Pelotas - Campus Capão do Leão - Pelotas, RS

\begin{abstract}
RESUMO
Determinou-se o limiar anaeróbico (V4) de 23 cavalos Crioulos hígidos em treinamento. Os animais selecionados foram submetidos a testes de desempenho, composto por três etapas de velocidade progressiva de $6 \mathrm{~m} / \mathrm{s}, 8 \mathrm{~m} / \mathrm{s}$ e $10 \mathrm{~m} / \mathrm{s}$, com duração de cinco minutos cada etapa, até que fosse atingida a concentração de $4 \mathrm{mmol} / \mathrm{dL}$ de lactato sanguíneo. As concentrações de lactato sanguíneo elevaram-se a partir da velocidade de exercício de $6,0 \mathrm{~m} / \mathrm{s}$, sendo que 20 animais $(90 \%)$ atingiram $4 \mathrm{mmol} / \mathrm{L}$ de lactato na velocidade entre $6-8 \mathrm{~m} / \mathrm{s}$, com média de frequência cardíaca que variou de 121 a $140 \mathrm{bpm}$. Concluiu-se que a V4 do cavalo Crioulo está entre as velocidades de $6-8 \mathrm{~m} / \mathrm{s}$ e a frequência cardíaca entre 121 $140 \mathrm{bpm}$.
\end{abstract}

Palavras-chave: cavalo Crioulo, limiar anaeróbico, exercício, lactato

\begin{abstract}
The anaerobic threshold (V4) while running on a track is used in many breeds to evaluate physical conditioning and to establish a training regimen, but these data have not been obtained for the Crioulo breed. This study evaluated 23 healthy Crioulo horses to determine the anaerobic threshold (V4) during training. The selected animals were subjected to a performance test on a track. The test consisted of three five minute stages with increasing speeds of $6 \mathrm{~m} / \mathrm{s}, 8 \mathrm{~m} / \mathrm{s}$ and $10 \mathrm{~m} / \mathrm{s}$ until the blood lactate concentration reached $4 \mathrm{mmol} / \mathrm{dL}$. Blood lactate concentrations began to increase at the exercise speed of $6 \mathrm{~m} / \mathrm{s}$, and $90 \%(n=20)$ of the animals reached $4 \mathrm{mmol} / \mathrm{dL}$ lactate between $6-8 \mathrm{~m} / \mathrm{s}$, with an average heart rate ranging from 121 to $140 \mathrm{bpm}$ at these speeds. Our results demonstrate that the V4 of the Crioulo horse is between $6-8 \mathrm{~m} / \mathrm{s}$ at a heart rate between 121 and $140 \mathrm{bpm}$.
\end{abstract}

Keywords: anaerobic threshold, Crioulo horse, exercise, lactate

\section{INTRODUÇÃO}

A Associação Brasileira de Criadores de Cavalos Crioulos (ABCCC) registra atualmente 339.924 animais, sendo a grande maioria localizada no estado do Rio Grande do Sul. O estado é considerado pioneiro na criação e valorização da raça no país. Inicialmente os animais eram utilizados para serviços em fazendas e manejo com gado, porém, há aproximadamente 27 anos, foi criada uma competição morfológica e funcional, denominada Freio de Ouro, a qual foi o marco da transição do cavalo Crioulo, até então um animal de trabalho, para o cavalo Crioulo atleta (Gianluppi et al., 2009). Essa transição conferiu à raça uma crescente valorização econômica, no entanto, cientificamente, pouco se conhece sobre seu perfil atlético durante o exercício.

A prova Freio de Ouro é dividida em duas etapas, uma morfológica e outra funcional. $\mathrm{Na}$ etapa funcional, são praticadas as provas de andadura, figura, esbarro e giro sobre patas, aparte e "pechada" no gado e, por fim, paleteada (Associação..., 2010), e os tempos médios desenvolvidos são de 60s, 82s, 19s, 132s e $23 \mathrm{~s}$ para cada prova, respectivamente. As

Recebido em 20 de maio de 2011

Aceito em 6 de novembro de 2012

E-mail: lo1amaral@gmail.com 
velocidades médias percorridas para completar cada fase da prova funcional são de $8 \mathrm{~m} / \mathrm{s}$ na andadura, $4,6 \mathrm{~m} / \mathrm{s}$ na figura e $9,4 \mathrm{~m} / \mathrm{s}$ na paleteada. A competição é disputada durante três dias e é caracterizada pela exigência de velocidade e resistência dos animais (Amaral, 2011). O desgaste físico e o curto período de recuperação requerem aptidão funcional e capacidade física para desempenhar o exercício. A seleção de animais por aptidão funcional valorizou de forma expressiva o condicionamento físico ideal e a utilização de protocolos de treinamento adequados para cada animal (Gianluppi et al., 2009).

Varias técnicas são descritas para avaliação da condição física de equinos, no entanto os testes realizados a campo (pista) são a melhor forma de se obterem dados específicos de cada indivíduo. Esses testes têm como base a utilização do controle de frequência cardíaca (FC) e dosagem sanguínea de lactato. A relação $\mathrm{FC} x$ lactato fornece dados que possibilitam tanto a avaliação do condicionamento físico como a prescrição da intensidade de treinamento, tornando-se uma valiosa ferramenta para maximização dos resultados obtidos nas competições (Erck et al., 2007).

A análise da concentração de lactato sanguíneo, em situações específicas de exercício, tem sido sugerida como um meio de se obterem informações relevantes a respeito da característica metabólica do atleta (Erickson et al., 1991). Esse fato é justificado pela relação positiva entre concentração do lactato sanguíneo e intensidade de exercício, sendo a variável que apresenta a melhor correlação com a performance competitiva do animal. A correlação é determinada pela V4, ou limiar anaeróbico, conceituada como a velocidade em que ocorre o equilíbrio entre a produção e o consumo do lactato. No equino, esse limiar é conhecido quando o indivíduo atinge $4 \mathrm{mmol} / \mathrm{L}$ de lactato sanguíneo. Animais que desenvolvem velocidades superiores à sua V4 têm seu metabolismo energético predominantemente anaeróbico, o que acumula lactato e confere fadiga muscular (Lindner e Boffi, 2006).

A V4 é uma técnica difundida mundialmente na maioria das raças de esporte. Apesar de especulações, dentro da raça Crioula não existem dados concretos sobre os parâmetros metabólicos desses animais. O conhecimento da V4 fornece parâmetros fisiológicos específicos de cada indivíduo. Portanto, o programa de treinamento deixa de ser realizado somente de maneira empírica, tornando-se um processo técnico, com embasamento clínico e metabólico (Vonwittke et al., 1994; Lindner et al., 2000; Erck et al., 2007).

O objetivo deste trabalho foi determinar a V4 de cavalos Crioulos em treinamento, relacionandose esse valor com a frequência cardíaca alcançada em diferentes velocidades.

\section{MATERIAL E MÉTODOS}

Os testes foram realizados em cinco centros de treinamento de cavalos Crioulos, localizados nas cidades de Bagé, Rio Grande, Uruguaiana, Porto Alegre e Arambaré, no estado do RS. Os animais envolvidos realizavam treinamento para prova funcional do Freio de Ouro em pistas gramadas e planas. O estudo foi dividido em duas etapas: seleção dos animais e determinação da V4.

Para seleção, 32 animais foram avaliados clinicamente por meio da determinação de frequência cardíaca, frequência respiratória, tempo de perfusão capilar, coloração de mucosas e temperatura corpórea, além das análises sanguíneas de hematócrito, hemoglobina, proteína plasmática total, fibrinogênio e leucograma. Essa etapa tinha como objetivo categorizar e selecionar animais hígidos com semelhante padrão de condicionamento. O processo foi realizado conforme Marlin e Nankervis (2002), os quais determinam que animais com condicionamento físico adequado para desempenhar uma determinada atividade apresentam a frequência cardíaca inferior a $100 \mathrm{bpm}$, dois minutos após o exercício.

Com base nesse conceito, os animais foram submetidos a um exercício em pista em velocidade de $8 \mathrm{~m} / \mathrm{s}$ durante cinco minutos, com o objetivo de atingirem frequências cardíacas superiores a 100bpm. A velocidade foi monitorada pelo cavaleiro por meio do GPS portátil (Forerunner® 405, Garmin). Foi avaliado o padrão cardíaco em repouso, imediatamente após o exercício e dois minutos após o exercício. Foram considerados aptos ao estudo os animais nos quais a frequência cardíaca, dois minutos após o exercício, fosse inferior a 100bpm. Com base nesses resultados, foram selecionados 23 
equinos hígidos, sendo 12 machos inteiros e 11 fêmeas, com idade média de $6 \pm 2$ anos, peso corporal médio de $420 \pm 50 \mathrm{~kg}$ e com adequado condicionamento físico.

$\mathrm{Na}$ segunda etapa do estudo, foi efetuada a avaliação do limiar anaeróbico (V4), na qual os animais selecionados, como descrito acima, foram submetidos a testes progressivos de desempenho em pista circular gramada de $160 \mathrm{~m}$. Antes do teste, os animais desempenharam um exercício a trote por 10 minutos, como forma de elevar a temperatura corporal para prevenir lesões.

O teste consistiu em escalas de exercício de cinco minutos, num total de três escalas, intervaladas por um período de três minutos para coleta de sangue e aferição da frequência cardíaca, conforme preconizado por Lindner (2000).

As velocidades foram categorizadas em momentos (M), sendo repouso inicial (M1), velocidade de $6 \mathrm{~m} / \mathrm{s}$ (M2), velocidade de $8 \mathrm{~m} / \mathrm{s}$ (M3), velocidade de $10 \mathrm{~m} / \mathrm{s}$ (M4) e repouso 25 minutos após o exercício (M5). O cavaleiro monitorou as velocidades por meio de GPS portátil (Forerunner® 405, Garmin, EUA).

O controle cardíaco foi efetuado pela aferição da FC com estetoscópio no momento de repouso (M1), após cada escala de velocidade e 25 minutos após a última escala (M5). Os animais foram categorizados de acordo com a frequência cardíaca, em escalas de até 60bpm, de 61120bpm, 121-140bpm e acima de 140bpm. A determinação da V4 foi efetuada pela velocidade na qual os animais atingissem $4 \mathrm{mmoL} / \mathrm{L}$ de lactato sanguíneo. Assim, foram colhidas amostras de sangue venoso em repouso, imediatamente após cada escala de velocidade e 25 minutos após a última velocidade desenvolvida pelo animal. $O$ teste foi considerado concluído quando os animais atingissem 4mmoL/L de lactato sanguíneo (V4). As coletas foram efetuadas em tubos de vácuo (Vacutainer ®, EUA) contendo fluoreto de potássio. A análise foi realizada imediatamente após a coleta, por meio de equipamento portátil de dosagem por fitas reagentes (Lactímetro Portátil - Accutrend Plus, Roche®, Alemanha).
A análise dos resultados foi realizada utilizando-se o programa Statistix ${ }^{\hat{a}}$ (2003). Foram comparadas a concentração de lactato e a FC nas diferentes velocidades. Ainda, a FC foi categorizada, de forma a determinar a relação objetiva com a concentração de lactato. Para todas as comparações foi utilizada análise de variância seguida de comparação de médias pelo teste de Tukey HSD $(\mathrm{P}<0,05)$. Para predizer o efeito da velocidade sobre a frequência cardíaca e sobre o lactato sanguíneo, foi utilizada a análise de regressão polinomial, sendo os modelos escolhidos de acordo com a significância dos coeficientes de regressão $(\mathrm{P}<0,05)$ e pelo coeficiente de determinação. $\mathrm{O}$ teste de Pearson foi realizado para determinar o tipo de relação das análises de FC e lactato sanguíneo.

Este estudo foi aprovado pelo Comitê de Ética e Experimentação Animal no 4602/2010.

\section{RESULTADOS}

Por meio do teste desenvolvido na primeira etapa do estudo, foi possível selecionar animais hígidos em semelhante condição física para a mensuração da V4 na segunda etapa. Nesta etapa, conforme demonstrado na Tab. 1, foram determinados os valores médios de FC e lactato dos animais em repouso, em carga progressiva de exercício e 25 minutos após o esforço.

Nas frequências cardíacas de até $60 \mathrm{bpm}$, de 61 120bpm, 121-140bpm e acima de 140 bpm, os valores médios ( \pm erro-padrão da média) de lactato foram de $1,16(0,43), 2,31(0,56), 5,56$ $(0,78)$ e $10,07(0,78) \mathrm{mmol} / \mathrm{L}$, respectivamente. Não houve diferença $(\mathrm{P}>0,05)$ entre os valores de lactato até $120 \mathrm{bpm}$. Porém, nas frequências cardíacas de 120-140bpm e acima de 140bpm, ocorreu diferença $(\mathrm{P}<0,05)$ entre elas.

A média de lactato sanguíneo em repouso (M1) foi de $0,75 \mathrm{mmol} / \mathrm{L}$. Após a primeira escala de velocidade de $6 \mathrm{~m} / \mathrm{s}$ (M2), a média de lactato elevou-se para 2,29mmol/L, sendo que nesta velocidade quatro animais (18\%) atingiram o valor de $4 \mathrm{mmol} / \mathrm{L}$ de lactato sanguíneo. Após a velocidade de $8 \mathrm{~m} / \mathrm{s}$ (M3), observou-se aumento dos valores de lactato, com média de $8,88 \mathrm{mmol} / \mathrm{L}$. Nesta velocidade, 15 animais $(68 \%)$ ultrapassaram o valor de $4 \mathrm{mmol} / \mathrm{L}$ e um $(4,5 \%)$ atingiu $4 \mathrm{mmol} / \mathrm{L}$, sendo observado valor 
máximo de $17 \mathrm{mmol} / \mathrm{L}$ e valor mínimo de $1,7 \mathrm{mmol} / \mathrm{L}$. A velocidade de $10 \mathrm{~m} / \mathrm{s}$ (M4) foi desenvolvida por dois cavalos (9\%), que apresentaram média de $10,75 \mathrm{mmol} / \mathrm{L}$ de lactato sanguíneo. Os valores de lactato sanguíneo foram maiores em M3 e M4 (P<0,05), em comparação aos outros momentos, conforme a Tab. 1. Ainda, quando avaliados o M1 e o M5, não houve diferença $(\mathrm{P}>0,05)$ nos valores de lactato, o que demonstra que, em média, nos animais em M5 ocorreu desvio para o comportamento basal (M1).

Tabela 1. Valores médios ( \pm erro-padrão da média) de frequência cardíaca e lactato de equinos em diferentes velocidades

\begin{tabular}{cccc}
\hline Velocidade & $\mathrm{N}$ & Frequência cardíaca $(\mathrm{bpm})$ & Lactato $(\mathrm{mmol} / \mathrm{L})$ \\
\hline Repouso* & 22 & $47,45(2,86) \mathrm{c}$ & $0,75(0,54) \mathrm{b}$ \\
$6 \mathrm{~m} / \mathrm{s}$ & 22 & $116,36(2,86) \mathrm{b}$ & $2,29(0,54) \mathrm{b}$ \\
$8 \mathrm{~m} / \mathrm{s}$ & 18 & $146,67(3,17) \mathrm{a}$ & $8,88(0,60) \mathrm{a}$ \\
$10 \mathrm{~m} / \mathrm{s}$ & 2 & $138,00(2,86) \mathrm{ab}$ & $10,75(0,54) \mathrm{a}$ \\
Repouso** $^{* *}$ & 22 & $53,09(9,51) \mathrm{c}$ & $1,71(1,80) \mathrm{b}$ \\
\hline
\end{tabular}

Médias seguidas por letras distintas na mesma coluna diferem entre si $(\mathrm{P}<0,05)$.

*Antes do início do exercício; **25 min após o término do exercício.

Em M5, 17 animais (77\%) retornaram o lactato sanguíneo aos valores de referência, $0,5-1,5 \mathrm{mmol} / \mathrm{L}$, enquanto os outros cinco (23\%) ainda apresentaram valores acima dos parâmetros fisiológicos No entanto, não houve diferença (Tab. 1) entre M1 e M5 (P>0,05). Foi observado que 20 animais $(90 \%)$ atingiram $4 \mathrm{mmol} / \mathrm{L}$ de lactato à velocidade de $6-8 \mathrm{~m} / \mathrm{s}$, e FC de 121-140bpm.
Observou-se a correlação positiva $\left(R^{2}=0.4912\right)$ entre frequência cardíaca e lactato sanguíneo $(\mathrm{P}<0,0001$; Fig. 1).

$\mathrm{Na}$ avaliação de regressão polinomial, observouse efeito quadrático tanto da concentração de lactato (Fig. 2) quanto da FC (Fig. 3), para as diferentes velocidades.

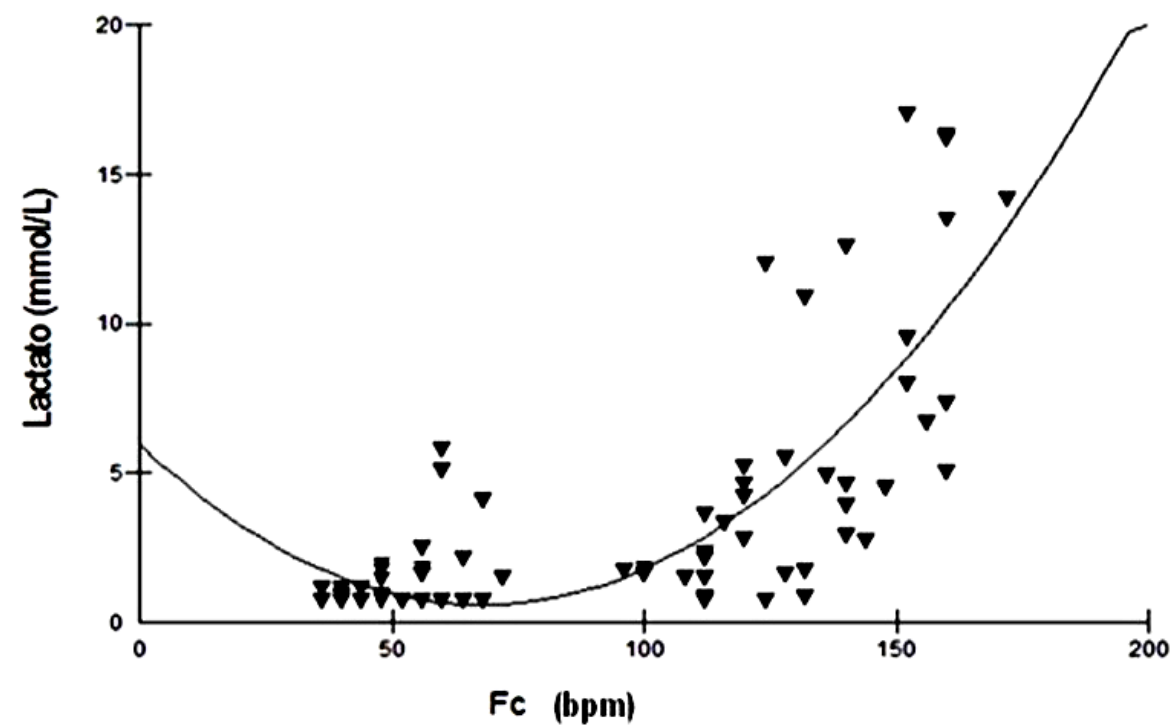

Figura 1. Correlação $\left(R^{2}=0,4912\right)$ entre frequência cardiaca e lactato sanguíneo observada no teste de Pearson, em cavalo Crioulo. 


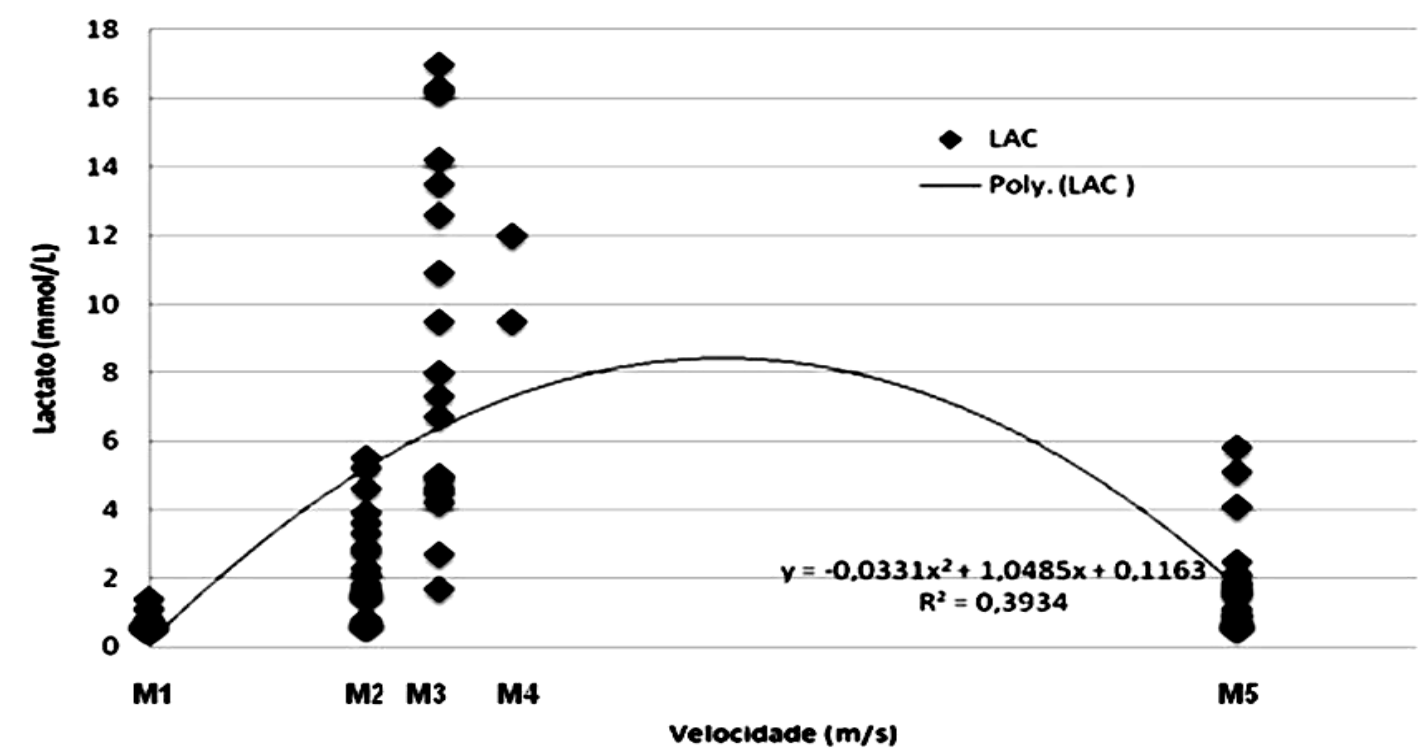

Figura 2. Teste de regressão polinomial demonstrando efeito quadrático da velocidade sobre a concentração de lactato nos distintos momentos (M), em cavalo Crioulo.

M1: repouso; M2: 6m/s; M3: 8m/s; M4: 10m/s e M5: 25 min após o término do exercício.

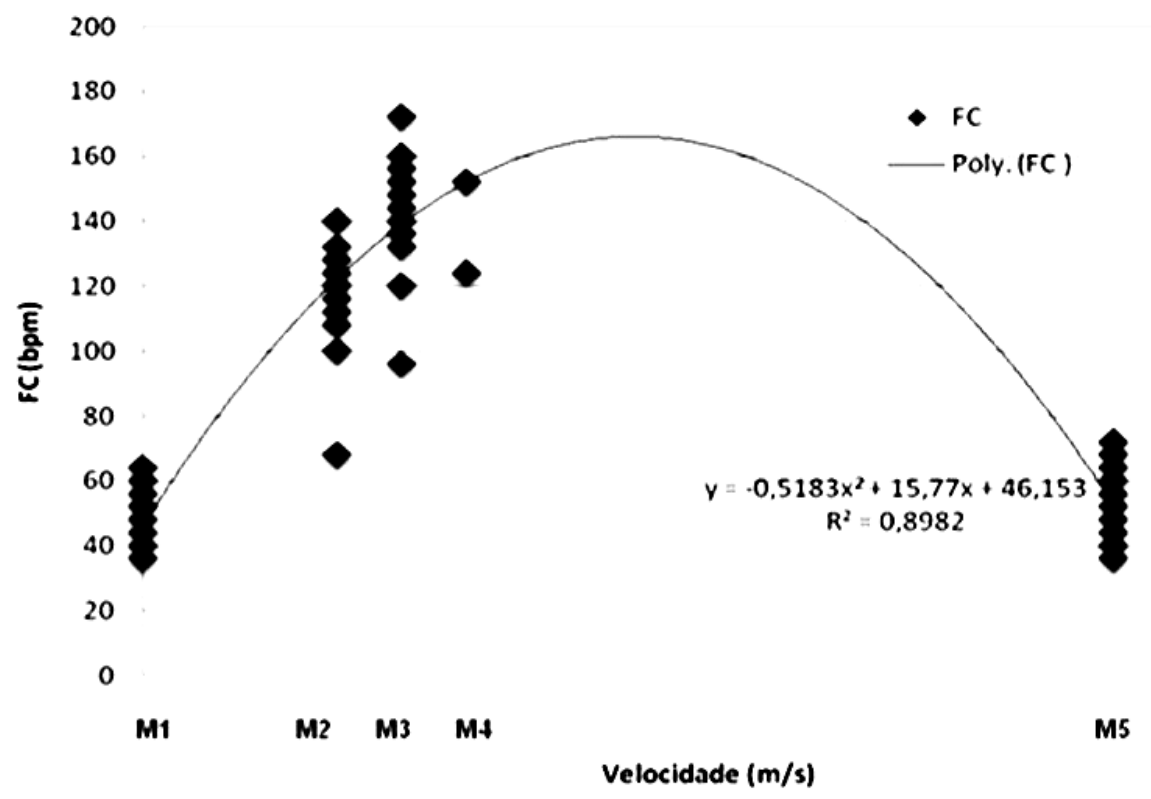

Figura 3. Teste de regressão polinomial demonstrando efeito quadrático da velocidade sobre a frequência cardíaca nos distintos momentos (M), em cavalo Crioulo.

M1: repouso; M2: $6 \mathrm{~m} / \mathrm{s} ; \mathrm{M} 3: 8 \mathrm{~m} / \mathrm{s} ; \mathrm{M} 4: 10 \mathrm{~m} / \mathrm{s}$ e M5: $25 \mathrm{~min}$ após o término do exercício.

Não houve diferença entre as velocidades M3 e M4 com relação à FC dos animais, porém este resultado pode ter sido influenciado pelo pequeno número de animais $(\mathrm{n}=2)$ avaliados na velocidade M4 em consequência de a maior parte dos equinos avaliados $(n=21)$ atingir a V4 entre as velocidades de M2 e M3. 


\section{DISCUSSÃO}

A mensuração da V4 obtida no presente estudo consiste em um passo importante para determinar e avaliar eficiência de protocolos de treinamento e condicionamento físico do cavalo Crioulo. $\mathrm{O}$ método de avaliação utilizado neste modelo experimental foi o mesmo descrito por Lindner (2000), o qual se demonstrou prático e eficiente para obtenção dos dados, visto que reproduz as condições de pista e treinamento aos quais os animais são submetidos durante as competições.

As FC observadas em M1, M2 e M3 foram diferentes $(\mathrm{P}<0,05)$ e maiores de acordo com o aumento da velocidade. Esse dado foi descrito em cavalos Puro Sangue Inglês em estudo realizado por Banister e Purvis (1968), no qual foi utilizada a eletrocardiografia para demonstrar a relação positiva entre velocidade e frequência cardíaca nos animais atletas.

Foi observado, a partir dos valores de FC e lactato, que a V4 do cavalo Crioulo ocorre em frequências que variam de 121-140bpm. Esse dado torna-se relevante para realização de protocolos de treinamento baseados no controle de frequência cardíaca, tendo conhecimento de que, em média, com valores de frequência acima de 120bpm, o animal estará em exercício predominantemente anaeróbico. No entanto, os dados sofrem variação de acordo com a raça e o condicionamento físico dos animais, como observado no estudo de Foreman et al. (1990), em equinos da raça Puro Sangue Inglês, no qual a V4 ocorreu em FC de 160 a 200bpm.

Com relação às avaliações de lactato sanguíneo, neste modelo experimental, foi observada média de lactato em M1 de $0,75 \mathrm{mmol} / \mathrm{L}$, não havendo diferença deste para M2, em que a média foi de $2,29 \mathrm{mmol} / \mathrm{L}$, o que demonstra que nessa velocidade e condição de avaliação o exercício permaneceu predominantemente aeróbico. Sendo assim, a cinética do lactato durante o teste progressivo por estágios apontou para um discreto aumento inicial, e depois, à medida que o exercício progrediu, ocorreu um aumento significativo na produção de lactato.

Segundo Evans et al. (2000), pequenos aumentos na concentração de lactato são observados com a progressão da velocidade de exercício, e para velocidades altas observa-se uma elevação exponencial das concentrações, o que caracteriza uma taxa de produção maior do que seu consumo pela via energética, redundando, assim, em acúmulo e fadiga, resultado que está de acordo com os obtidos neste estudo e com Lopes et al. (2009), os quais avaliaram cavalos em competição de vaquejada e observaram um aumento de $130 \%$ nos níveis de lactato após a prova, dado que caracteriza a intensa exigência física da modalidade. Em recente estudo com cavalos da raça Quarto de Milha usados em provas de Laço em Dupla, Caiado et al. (2011) observaram aumento significativo na concentração de lactato e creatinoquinase imediatamente após a competição, enquanto as concentrações de aspartato aminotransferase não sofreram modificação. A avaliação desses dados permitiu concluir que os animais estavam aptos ao nível de exercício imposto.

De acordo com o teste de Pearson, o lactato sanguíneo foi proporcional ao aumento de velocidade, principalmente entre $6 \mathrm{~m} / \mathrm{s}$ e $8 \mathrm{~m} / \mathrm{s}$, velocidades em que a média desse metabólito foi diferente. $\mathrm{O}$ conhecimento desse dado demonstra que o limite do metabolismo predominantemente aeróbico está nas velocidades entre $6 \mathrm{~m} / \mathrm{s}$ e $8 \mathrm{~m} / \mathrm{s}$ e permite determinar protocolos de treinamento conforme a exigência do animal em pista de competição.

A média da FC e a do lactato foram proporcionais à intensidade de exercício. Isto é justificado pelo fato de que, no organismo, à medida que aumentam as necessidades energéticas e de oxigênio durante o exercício, o sistema cardiorrespiratório responde aumentando as frequências cardíaca e respiratória de forma a disponibilizar maior volume sanguíneo oxigenado ao tecido muscular e maior aporte energético. À proporção que a intensidade do esforço aumenta, o organismo chega ao seu ponto máximo de disponibilização de energia e $\mathrm{O} 2$, passando a aumentar também a concentração de lactato sanguíneo. Isto explica a relação positiva que existe entre a intensidade de exercício, FC e concentração de lactato (Lindner e Boffi, 2006).

As médias de FC e os valores de lactato em M1 e M5 não diferiram entre si. Segundo Engelhardt (1977), Cabezas et al. (1994) e Evans (2000), os valores de lactato sanguíneo após exercício devem retornar aos padrões fisiológicos em uma 
hora e a FC deve retornar ao valor inicial em 2530 min. Esse tempo de retorno está relacionado ao período em que o lactato é transportado para fora da célula muscular, atinge a corrente sanguínea e é reutilizado na forma de glicose por outros tecidos (ciclo de Cori). Tal processo demanda energia e oxigênio, por isso, após o término do exercício, as frequências cardíaca e respiratória permanecem altas, de modo a manter a oxigenação adequada (Gonzáles, 2006).

Na prova do Freio de Ouro, os cavalos são submetidos a exigências tanto de resistência quanto de velocidade, competem durante três dias e desenvolvem velocidades acima de $11 \mathrm{~m} / \mathrm{s}$ (Amaral, 2011). Dessa forma, necessitam de condicionamento físico adequado para desempenhar o exercício e evitar lesões. Neste experimento, foi observado que, mesmo todos os animais estando em treinamento para a prova do Freio de Ouro, apenas três deles $(13,5 \%)$ apresentaram a V4 acima de $8 \mathrm{~m} / \mathrm{s}$, o que sugere que grande parte dos animais compete sem condicionamento físico adequado, executando as etapas da prova com predominância do metabolismo anaeróbico e acúmulo de lactato sanguíneo. Essa observação também foi feita por Perez et al. (1997), que avaliaram cavalos Crioulos Chilenos em treinamento e em prova e constataram que os animais eram submetidos no treinamento a esforço físico inferior ao desempenhado em provas funcionais nas quais atingiam velocidade média de até $8 \mathrm{~m} / \mathrm{s}$ e concentração sanguínea de lactato de até 15 $\mathrm{mmol} / \mathrm{L}$. Sendo assim, estes dados questionam o modo como são realizados os protocolos de treinamento do cavalo Crioulo para prova funcional. No entanto, são necessários mais estudos com relação à caracterização da exigência física da prova para que se possa afirmar essa hipótese.

\section{CONCLUSÃO}

O limiar anaeróbico (V4) do cavalo Crioulo está entre as velocidades de $6-8 \mathrm{~m} / \mathrm{s}$ e frequência cardíaca entre 121 - 140bpm.

\section{REFERÊNCIAS}

AMARAL, L.A. Caracterização funcional da competição equestre "Freio de Ouro" segundo velocidade e tempo de exercício. Local, 2011. Pagina. Não publicado.

ASSOCIAÇÃO Brasileira de Criadores de Cavalos Crioulos. ABCCC. Sobre a prova Freio de Ouro. Disponível em: $</$ www.freiodeouro.com.br/historia $>$. Acesso em 15 de outubro de 2010

BANISTER, E.W.; PURVIS, A.D. Exercise electrocardiography in the horse by radiotelemetry. J. Am. Vet Med. As., v.152, p.1004-1008, 1968.

CABEZAS, I.S.; VALENZUELA, V.; MERINO, $\mathrm{J}$. et al. Rendimiento físico, requerimientos energeicos y adaptación fisiológica del caballo de tiro en faenas de aradura. Arch. Med. Vet. v.26, p.15-27, 1994.

CAIADO, J.C.C.; PISSINATE, G.L.; SOUZA, V.R.C. et al. Lactacidemia e concentrações séricas de aspartato aminotransferase e creatinoquinase em equinos da raça Quarto de Milha usados em provas de laço em dupla. Pesq. Vet. Bras., v.31, p.452-458, 2011.

ENGELHARDT, W.V. Cardiovascular effects of exercise and training in horses. Ad. Vet. Scin. Comp. Med., v.21, p.173-205, 1977.

ERCK, E.V.; VOTION, D.M.; SERTEYN, D.; ART, T. Evaluation of oxygen consumption during field exercise tests in Standardbred trotters. Eq. Comp. Exer. Phys., v.4, p.43-49, 2007.

ERICKSON, H.H.; LUNDING, C.S.; ERICKSON, B.K.; COFFMAN, J.R. Indices of performance in the racing Quarter Horse. In: PERSSON, S.G.B.; LINDHOLM, A.; JEFFCOTT, L.B. (eds). Eq. Exer. Phys. ICEEP Publications, Davis, CA. p.41-46, 1991.

EVANS, D.L. Training and fitness in athletic horses. Barton, Camberra: Rural Industries Research and Development Corporation, 2000. p.64. 
FOREMAN, J.H.; BAYLY, W.M.; GRANT, B.D.; GOLLNICK, P.D. Standardized exercise test and daily heart rate responses of Thoroughbred undergoing conventional race training and detraining. Am. J. Vet. Res. v.51, p.914-920, 1990.

GIANLUPPI, L.D.F.; BORTOLI, E.C.; SCHVARZ SOBRINHO, R. et al. Agregação de valor em equinos da raça Crioula: Um estudo de caso. Arch. Zoot., v.58, p.471-474, 2009.

GONZÁLES, G.O. Nutricion y alimentación del caballo atleta. In: BOFFI, F.M. Fisiología del ejercicio equino. Buenos Aires: InterMédica, 2006. p.209-218.

LINDNER, A. Use of blood biochemistry for positive performance diagnosis of sports horses in practice. Ver. Med. Vet., v.151, p.611-618, 2000.

LINDNER, A.E; BOFFI, F.M. Pruebas de ejercicio. In: BOFFI, F.M. Fisiología del ejercicio equino. Buenos Aires: InterMédica., 2006. p.146-153.
LOPES, K.; BATISTA, J.; DIAS, R.; SOTOBLANCO, B. Influência das competições de vaquejada sobre os parâmetros indicadores de estresse em equinos. Cienc. Anim. Bras., v.10, p.538-543, 2009.

MARLIN, D.; NANKERVIS, K. Equine Exercice Physiology. Madison: Blackwell Science, 2002. p.204.

PÉREZ, R.M.; GARCIA, I.; CABEZAS, R. et al. Actividad física y cambios cardiovasculares $\mathrm{y}$ bioquímicos del caballo chileno a la competencia de rodeo. Arch. Med. Vet., v.29, p.221-234, 1997.

STATISTIX. STATISTIX for Windows version 8.0. User's Manual. Analytical Software. Tallahassee, FL, USA. 2003.

VONWITTKE, P.; LINDNER, A.; DEEGEN, E.; SOMMER, H. Effects of training on blood lactate running speed relationship in Thoroughbred racehorses. J. Appl. Phys. v.77, p.298-302, 1994. 